\title{
Computational analysis of the interaction between transcription factors and the predicted secreted proteome of the yeast Kluyveromyces lactis Otávio JB Brustolini ${ }^{1}$, Luciano G Fietto ${ }^{2}$, Cosme D Cruz ${ }^{3}$ and Flávia ML Passos*1
}

Address: ${ }^{1}$ Departamento de Microbiologia, Instituto de Biotecnologia Aplicada à Agropecuária (BIOAGRO), Universidade Federal de Viçosa, Viçosa, MG, Brazil, ²Departamento de Bioquímica e Biologia Molecular, Instituto de Biotecnologia Aplicada à Agropecuária (BIOAGRO), Universidade Federal de Viçosa,Viçosa, MG, Brazil and ${ }^{3}$ Departamento de Biologia Geral, Instituto de Biotecnologia Aplicada à Agropecuária (BIOAGRO), Universidade Federal de Viçosa, Viçosa, MG, Brazil

Email: Otávio JB Brustolini - tavinbio@yahoo.com.br; Luciano G Fietto - lgfietto@ufv.br; Cosme D Cruz - cdcruz@ufv.br; Flávia ML Passos* - flpassos@ufv.br

* Corresponding author

Published: 25 June 2009

BMC Bioinformatics 2009, 10:194 doi:10.1186/1471-2105-10-194
Received: 18 November 2008

Accepted: 25 June 2009

This article is available from: http://www.biomedcentral.com/I47I-2/05/I0/194

(c) 2009 Brustolini et al; licensee BioMed Central Ltd.

This is an Open Access article distributed under the terms of the Creative Commons Attribution License (http://creativecommons.org/licenses/by/2.0), which permits unrestricted use, distribution, and reproduction in any medium, provided the original work is properly cited.

\begin{abstract}
Background: Protein secretion is a cell translocation process of major biological and technological significance. The secretion and downstream processing of proteins by recombinant cells is of great commercial interest. The yeast Kluyveromyces lactis is considered a promising host for heterologous protein production. Because yeasts naturally do not secrete as many proteins as filamentous fungi, they can produce secreted recombinant proteins with few contaminants in the medium. An ideal system to address the secretion of a desired protein could be exploited among the native proteins in certain physiological conditions. By applying algorithms to the completed $K$. lactis genome sequence, such a system could be selected. To this end, we predicted protein subcellular locations and correlated the resulting extracellular secretome with the transcription factors that modulate the cellular response to a particular environmental stimulus.

Results: To explore the potential Kluyveromyces lactis extracellular secretome, four computational prediction algorithms were applied to 5076 predicted $K$. lactis proteins from the genome database. SignalP v3 identified 418 proteins with $\mathrm{N}$-terminal signal peptides. From these 418 proteins, the Phobius algorithm predicted that 176 proteins have no transmembrane domains, and the big-PI Predictor identified 150 proteins as having no glycosylphosphatidylinositol (GPI) modification sites. WoLF PSORT predicted that the $K$. lactis secretome consists of 109 putative proteins, excluding subcellular targeting. The transcription regulators of the putative extracellular proteins were investigated by searching for DNA binding sites in their putative promoters. The conditions to favor expression were obtained by searching Gene Ontology terms and using graph theory.

Conclusion: A public database of $K$. lactis secreted proteins and their transcription factors are presented. It consists of I09 ORFs and 23 transcription factors. A graph created from this database shows I 34 nodes and 884 edges, suggesting a vast number of relationships to be validated experimentally. Most of the transcription factors are related to responses to stress such as drug, acid and heat resistance, as well as nitrogen limitation, and may be useful for inducing maximal expression of potential extracellular proteins.
\end{abstract}




\section{Background}

The General Secretory Pathway (GSP) is a protein export process of major biological and technological significance. Cell communication, as well as intercellular signaling and growth during development in multicellular organisms depends on the secretion pathway. The export of a commercial protein into the extracellular medium by a recombinant cell can facilitate its downstream processing. The yeast Kluyveromyces lactis is considered a promising host for heterologous protein production. Because yeasts naturally do not secrete as many proteins as filamentous fungi, they can produce secreted recombinant proteins with few contaminants in the medium [1]. An ideal system for secreting a desired protein could be developed from analysis of the native proteins. The completed $K$. lactis genome sequence provides the tools to construct such a system [2]. As the genomes of several hemiascomycetes yeasts are now sequenced [3-5] and cross-comparison does not reveal significant differences, the prospect of discovering a potentially significant secreted protein using bioinformatics techniques is high [6-8]. In K. lactis, as in other eukaryotes, secreted proteins are typically recognized by the presence of an $\mathrm{N}$-terminal signal sequence to direct them to GSP [11]. Signal sequences usually have a well-characterized structure composed of a central hydrophobic core (h-region). This consists of an average of 6-15 amino acid (aa) residues that are flanked by hydrophilic $\mathrm{N}$ - and C-terminal regions. The h-region is important for correct targeting and membrane insertion of the peptide. At the polar C-terminal region, helix breaking often occurs because of proline and glycine residues and small uncharged residues at the -3 and -1 positions that determine the signal peptide cleavage site $[9,10]$. The polar N-terminal region is variable in length and frequently positively charged [11]. Although some proteins lacking N-terminal signal sequences reach the extracellular medium, the majority of soluble secreted proteins in $K$. lactis are likely to be transported via the GSP [1]. A wide variety of computational methods have been used to predict the subcellular localization of proteins [12]. The methods differ in the input data they demand and the techniques applied to make decisions or predictions about location. Once the input data type are fixed, the methods for making predictions are basically by two methods: the manual construction of explicit rules for localization prediction using current knowledge of sorting signals, or applying data-driven, machine-learning techniques (e.g., Neural Networks (NN) or Hidden Markov Models, (HMMs)) [12]. The latter automatically extracts decision rules from the sets of proteins with known location, without making any prior, detailed assumptions about the features of interest.

In addition to using direct algorithm analysis to predict extracellular proteins, the extracellular secretome can be analyzed through its possible transcription factors (TFs). TFs are part of the signal transduction pathway that modulates the cell metabolism in response to environmental stimuli [13]. The TFs that contain DNA binding motifs are the component of the signaling pathway that is closest to the level of the DNA. To a large degree, the combinatorial presence and absence of transcription factor binding sites (TFBSs) is responsible for gene regulation complexity [1417]. The identification of TFBSs has been used to infer regulatory networks for several different yeasts [18].

Using an algorithm approach, we proposed identifying extracellular protein candidates in the yeast $K$. lactis and determining TFBSs in the promoters of their genes. Analysis of the relationship to transcriptional regulators used the dataset of Bussereau et al [18], and putative promoter regions $1 \mathrm{~kb}$ upstream of the genes that encode the predicted extracellular proteins.

\section{Results}

\section{Prediction of K. lactis extracellular proteins}

A flowchart of the algorithms used to generate the database of potential extracellular K. lactis proteins is in Figure 1. Using the standard criteria of SignalP v3.0 [11], and the $\mathrm{NN}$ and HMM scores from $5076 \mathrm{~K}$. lactis open reading frames (ORFs), 698 ORFs containing consensus sequences for $\mathrm{N}$-terminal signal peptides and signal peptidase cleavage sites within 10-40 amino acid residues were predicted. When the 418 deduced proteins harboring $\mathrm{N}$ terminal signal peptides were submitted to the Phobius algorithm [19], only 242 were predicted to carry extra transmembrane domains, excluding the transmembrane domain of the signal peptide. The following analyses were conducted with the remaining 176 ORFs. To identify GPI modification sites, the ORFs were submitted to big-PI Predictor [20], with the results indicating that 150 ORFs contained a signal peptide, no transmembrane domain, and no GPI modification site. As some GSP proteins may be targeted to intracellular organelles rather than the extracellular medium, the algorithm WoLF PSORT [21] was used to detect conserved addresses to organelles. The outcome indicated $150 \mathrm{ORFs}$ predicted with extracellular addresses. Among these, 109 had the highest $k$-NN score ( $17.78 \pm 5.68)$ l the remaining 41 had lower $k$-NN scores ( $5.354 \pm 3.989)$ and were excluded, to increase the probability of selecting actual secreted proteins for further correlation with the transcription factor dataset.

Using a statistical approach, the first GPS criteria, the signal peptide, was tested against the following datasets: YEP (yeast extracellular proteins sequences), KLRS (K. lactis random sequences), the predicted extracellular proteins determined by WoLF PSORT, and EPMS dataset by Swain et al [22] (Figure 2). The YEP scores showed NN S/D greater than 0.66 and HMM around 0.8, whereas KLRS 


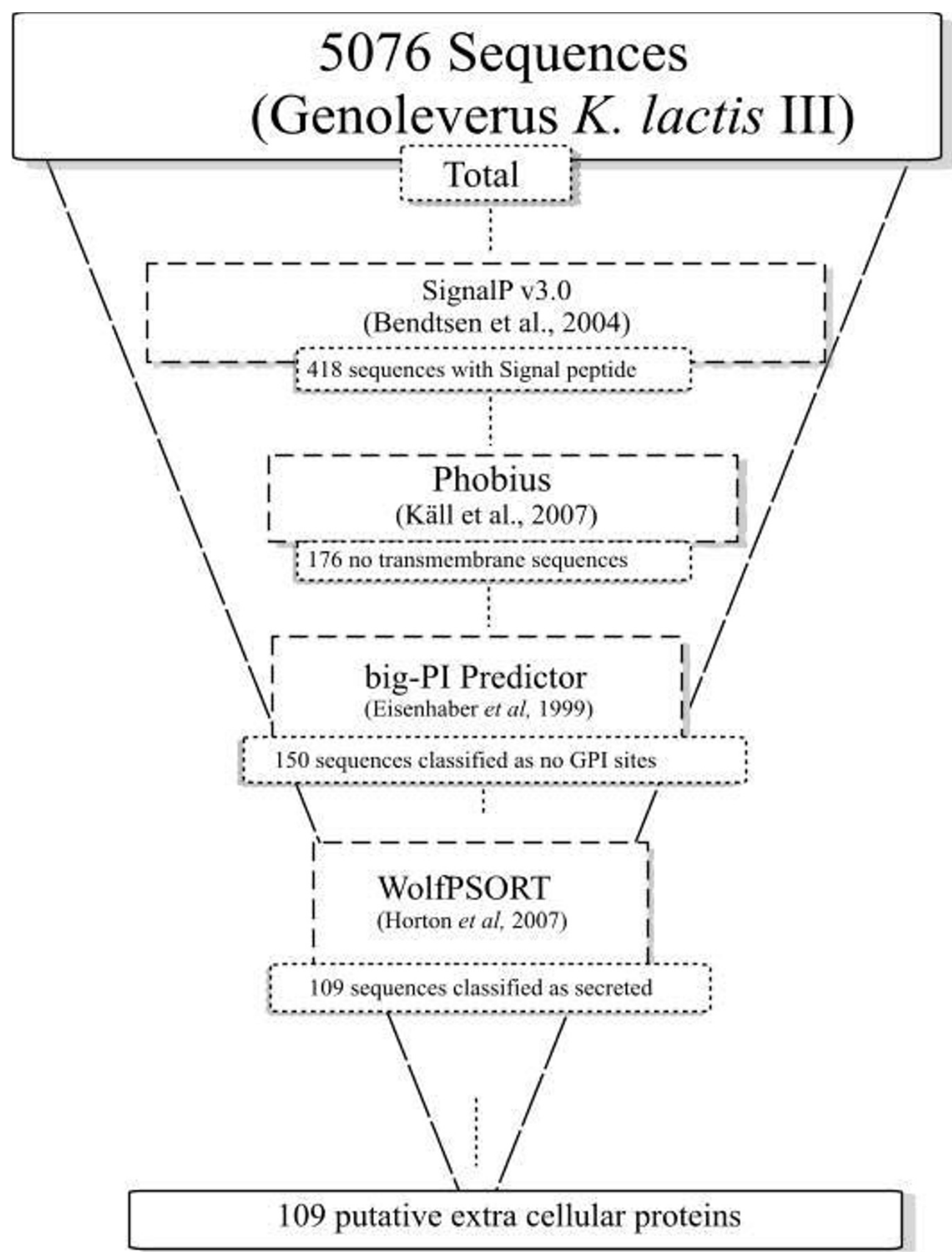

Figure I

Flowchart of the strategy adopted for mining $K$. lactis gene sequences for extracellular proteins and the outcome. 
A

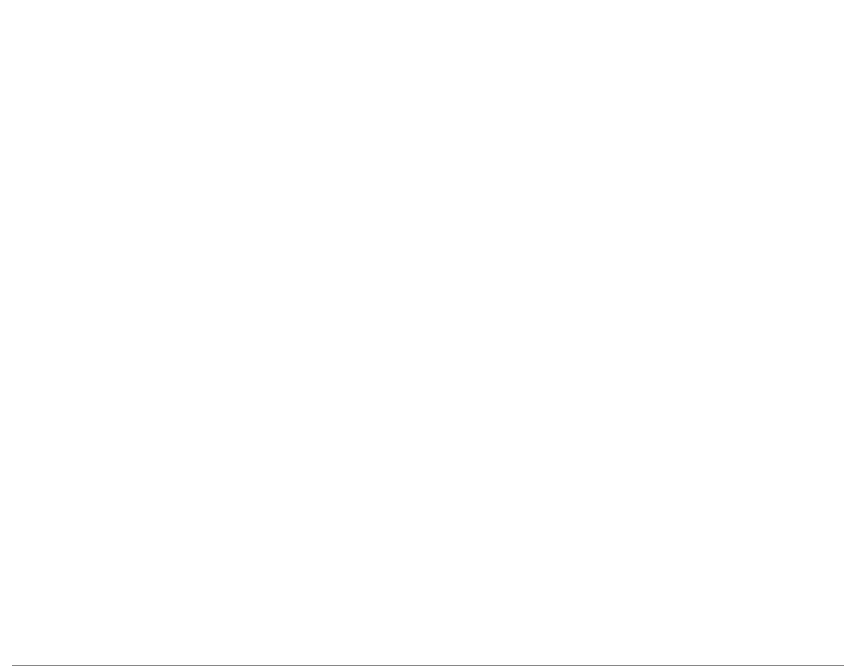

C

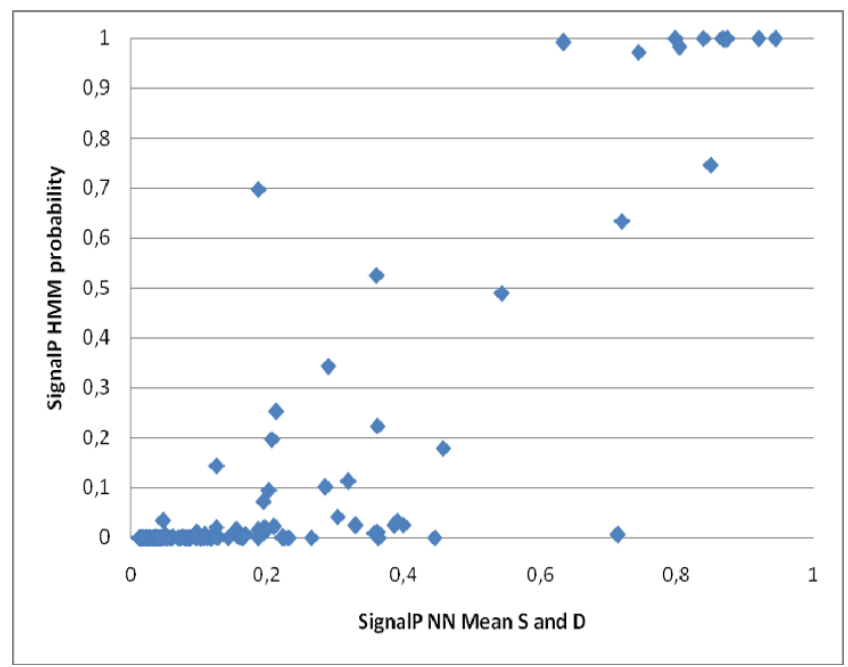

B

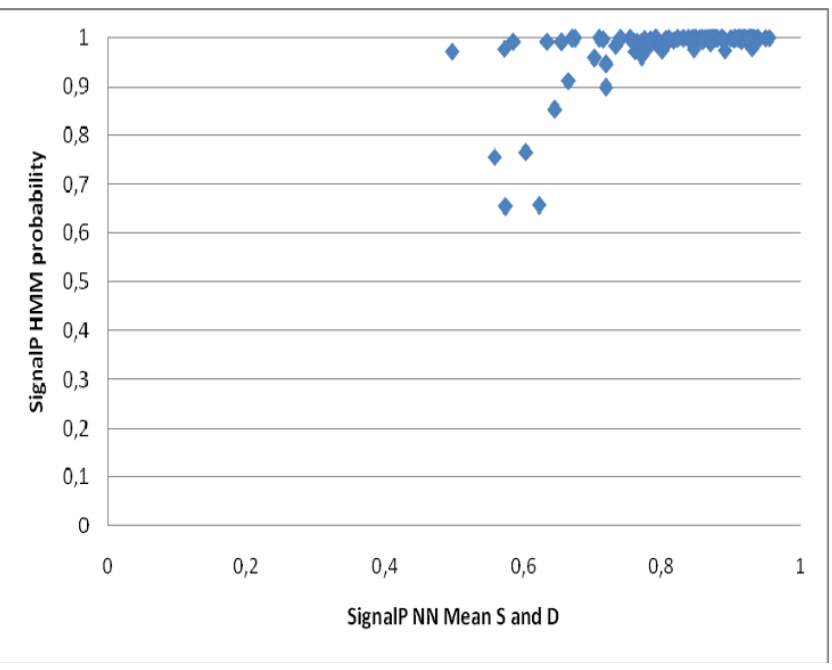

D

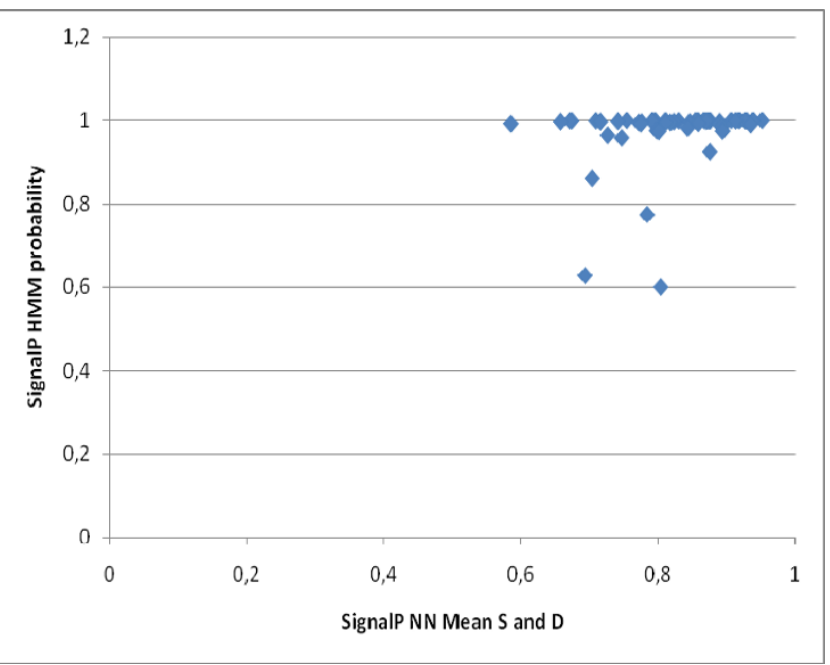

$\mathrm{E}$

\begin{tabular}{lcclcc}
\hline & \multicolumn{2}{c}{ Scores (Mean) } & \multicolumn{3}{c}{ Hotelling $\mathrm{T}^{2}$ Multivarite Test } \\
\hline Groups & SignalP - S and D & SignalP- HMM & Group Predicted vs & $\mathrm{T}^{2}$ & P-value \\
\hline Predicted & $0.8122 \pm 0.1012$ & $0.9410 \pm 0.1709$ & Annotated (YEP) & 0.1241 & 0.9401 \\
\hline Annotated (YEP) & $0.8387 \pm 0.0762$ & $0.9802 \pm 0.0572$ & Ramdom (KLRS) & 927.243 & $<0.01$ \\
\hline Random (KLRS) & $0.1516 \pm 0.2032$ & $0.0824 \pm 0.2431$ & EPMS (67\%) & 0.60695 & 0.741 \\
\hline EPMS (67\%) & $0.8251 \pm 0.0862$ & $0.9723 \pm 0.0799$ & & & \\
\hline
\end{tabular}

Figure 2

Analysis of the distribution of SignalP v3.0 scores: (A) 95 yeast extracellular proteins (YEP) dataset; (B) 109 K. lactis predict extracellular proteins; (C) $95 \mathrm{~K}$. lactis ramdom sequences (KLRS) from genome; (D) EPMS dataset from Swain et al [22] (E) Multivariate tests using Hotelling $\mathrm{T}^{2}$ to verify the statistical significance. 
simultaneously presented scores below 0.4 and 0.3 (Figs. $2 \mathrm{~A}$ and $2 \mathrm{C}$ ). The comparison between the controls for SignalP and the secreted ORFs scores revealed that the scores of the 109 ORFs were very similar to YEP, specifically, NN $\mathrm{S} / \mathrm{D}$ was 0.56 and HMM was 0.78 (Figure $2 \mathrm{~B}$ ). Thus, the standards criteria provided by SignalP were correctly encountered in all 95 sequences from the positive control. The EPMS dataset showed a high NN score $(>0.8)$ and high HMM score $(>0.75)$ in $67 \%$ of sequences (Figure 2D). Although the other 33\% were not detected as secreted by the predicted algorithms, the remaining $67 \%$ had a 0.74 probability of being equal to the predicted dataset according to T-square test (Figure 2E).

To evaluate the criteria for predicting the presence or absence of $\mathrm{N}$-terminal signal peptides in the K. lactis dataset, the Hotelling T-square multivariate test (Figure 2E) was employed on the basis of NN Mean S/D and HMM scores. The vector parameters for each control set were compared to the predicted set and confirmed by T-square test. The estimated 109 ORFs were closer to the YEP dataset $(\mathrm{p}=0.9401)$ than the KLRS $(\mathrm{p}<0.01)$.

\section{Analysis of annotations}

The biological significance of the predicted extracellular proteins of $K$ lactis was determined on the basis of annotations available at the Genolevures website http:// www.genolevures.org. Of the 109 predicted K. lactis extracellular proteins, 85 were annotated as similar to $S$. cerevisiae, and five as documented $K$. lactis proteins. Enzymes were the largest functional group $(48 \%)$ of known predicted proteins. A smaller group (4\%) was predicted as having a pheromone or mating-type function. Among the known sequences, 9\% were considered intracellular proteins or wrong predictions (Figure 3A). For those unknown potential $K$. lactis extracellular proteins (25\%), the Protein Family database (PFam) was applied to attempt to find relationships to known protein families through conserved domains (Figure $3 \mathrm{~B}$ ). The results dem- onstrated nine singletons among 21 that harbored conserved domains with varying PFam scores. The alpha mating factor precursor N-terminus (KLLA0A00154g, KLLA0F00220g), kappa casein (KLLA0B05731g), NADH dehydrogenase subunit 2 C-terminus (KLLA0C10054g), bacterial regulatory protein-Fis family (KLLA0D00660g), thioredoxin (KLLA0E05544g), mucin-like glycoprotein (KLLA0E10967g, KLLA0E19657g), and collagen triple helix repeat (KLLA0F01595g) all gave higher PFam scores. Analysis of the improbable secreted domains was carried out by alignment using BLAST tools http:// blast.ncbi.nlm.nih.gov/Blast.cgi. From nine sequences, six with nonsecreted domains were found to have a possible relation to extracellular proteins in other taxons.

\section{Relationship between the predicted extracellular proteins and transcriptional factors repertoire}

The putative promoter region was taken as one $\mathrm{kb}$ upstream of each predicted extracellular protein-encoding ORF and analyzed using the Yeastract website tool [23] to identify TFBSs related to $S$. cerevisiae. The results indicated the presence of 65 different TFBSs. In addition, the supporting algorithms in Supplementary Materials found 23 TFs homologs in K. lactis when comparing the K. lactis TFs dataset published by Bussereau et al [18] to S. cerevisiae. At least two TFBS in each promoter region were established by this analysis. In the Yeastract database, all the TFs have Gene Ontology (GO) terms http://www.geneontol ogy.org, that is, known details about the cellular function and address. These data (Table 1) showed that all 109 sequences have the TFBS for Mot3p (involved in repression of a subset of hypoxic genes and repression of ergosterol biosynthetic genes), 100 had a site for Stb5p (activator of multidrug resistance genes), 97 for Fkh1p (a minor role in the expression of G2/M-specific transcription in mitotic cell cycle), 45 for Gcn4p (activator of aa biosynthetic genes in response to aa starvation), 40 for Hac1p (regulates the unfolded protein response), 35 for Mcm1p (pheromone response), 33 for Rgt1p (regulates
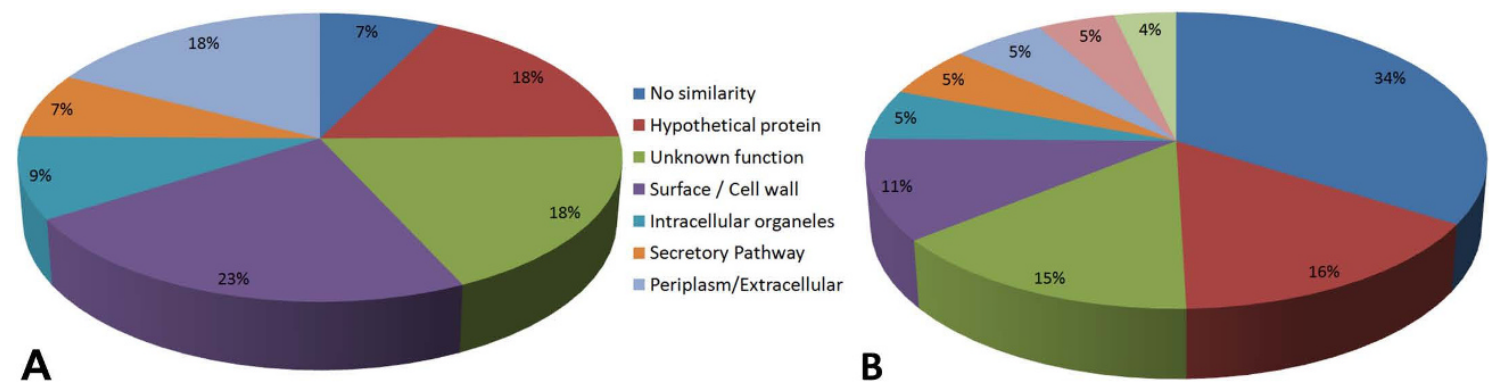

- Unknown

- Cell Wall /Surface

a Glyco-hydrolase

- Protease/Peptidase

acid Phosphatase

m Flocculin

॥ Secretory pathway

= Periplasm/extracellular

m Glucosidases

Figure 3

Characterization of the predicted proteins from (A) subcellular location in Génolevures annotation (release 3) and (B) by function and conserved domains in Protein Family (PFam 23.0). 
Table I: Cluster of transcription factors with GeneOntology terms related to the predicted ORFs

\begin{tabular}{|c|c|c|c|}
\hline Biological Process & T.F. & ORFs & Description Yeastract/GO \\
\hline \multirow[t]{2}{*}{ Aerobic/Anaerobic and Sterol metabolism } & Mot3p & 109 & $\begin{array}{l}\text { Repression of hypoxic genes, several DAN/TIR genes during aerobic growth, and } \\
\text { ergosterol biosynthetic genes }\end{array}$ \\
\hline & Hap4p & 2 & $\begin{array}{l}\text { Subunit of the heme-activated, glucose-repressed Hap } 2 p / 3 p / 4 p / 5 p \text { CCAAT-binding } \\
\text { complex, a transcriptional activator and global regulator of respiratory gene } \\
\text { expression; provides the principal activation function of the complex }\end{array}$ \\
\hline \multirow[t]{5}{*}{ Cell Cycle } & Fkhlp & 97 & $\begin{array}{l}\text { The expression of } G 2 / M \text { phase genes; negatively regulates transcriptional elongation; } \\
\text { positive role in chromatin silencing at } \mathrm{HML} \text { and } \mathrm{HMR} \text {. }\end{array}$ \\
\hline & Cbflp & II & Required for nucleosome positioning at this motif; targets Iswlp to DNA \\
\hline & Ace2p & 9 & $\begin{array}{l}\text { Activates expression of early GI-specific genes, localizes to daughter cell nuclei after } \\
\text { cytokinesis and delays GI progression in daughters. }\end{array}$ \\
\hline & RIm Ip & 5 & Maintenance of cell integrity; phosphorylated and activated by the MAP-kinase Slt2p \\
\hline & Swi5p & 9 & $\begin{array}{l}\text { Transcription factor that activates transcription of genes expressed at the M/GI } \\
\text { phase boundary and in GI phase }\end{array}$ \\
\hline \multirow[t]{3}{*}{ Drugs and metal resistance } & Stb5p & 100 & $\begin{array}{l}\text { Activator of multidrug resistance genes, forms a heterodimer with Pdrlp; interacts } \\
\text { with a PDRE (pleotropic drug resistance element) }\end{array}$ \\
\hline & Yaplp & 6 & $\begin{array}{l}\text { Required for oxidative stress tolerance; activated by } \mathrm{H} 2 \mathrm{O} 2 \text {; mediates resistance to } \\
\text { cadmium }\end{array}$ \\
\hline & Yrrlp & 7 & $\begin{array}{l}\text { Activates genes involved in multidrug resistance; paralog of Yrm lp, acting on an } \\
\text { overlapping set of target genes }\end{array}$ \\
\hline \multirow[t]{4}{*}{ General stress response } & Haclp & 40 & $\begin{array}{l}\text { Regulates the unfolded protein response, via UPRE binding, and membrane } \\
\text { biogenesis; ER stress-induced splicing pathway utilizing Ire Ip, TrlIp and Ada5p } \\
\text { facilitates efficient Haclp synthesis }\end{array}$ \\
\hline & Gis Ip & 20 & $\begin{array}{l}\text { JmjC domain-containing histone demethylase; transcription factor involved in the } \\
\text { expression of genes during nutrient limitation; also involved in the negative } \\
\text { regulation of DPPI and PHRI }\end{array}$ \\
\hline & Msn2p & 17 & $\begin{array}{l}\text { Transcriptional activator related to Msn4p; activated in stress conditions, which } \\
\text { results in translocation from the cytoplasm to the nucleus; binds DNA at stress } \\
\text { response elements of responsive genes, inducing gene expression }\end{array}$ \\
\hline & $\operatorname{Rtg} 3 p$ & 82 & $\begin{array}{l}\text { Basic helix-loop-helix-leucine zipper (bHLH/Zip) transcription factor that forms a } \\
\text { complex with another bHLH/Zip protein, Rtglp, to activate the retrograde (RTG) } \\
\text { and TOR pathways }(I, 2)\end{array}$ \\
\hline Pheromone response & Mcm Ip & 35 & $\begin{array}{l}\text { Involved in cell-type-specific transcription and pheromone response; plays a central } \\
\text { role in the formation of both repressor and activator complexes. }\end{array}$ \\
\hline \multirow[t]{2}{*}{ Amino acid/Nitrogen starvation response } & Gcn4p & 45 & $\begin{array}{l}\text { Amino acid biosynthetic genes in response to amino acid starvation; expression is } \\
\text { tightly regulated at both the transcriptional and translational levels }\end{array}$ \\
\hline & Met4p & 6 & $\begin{array}{l}\text { Responsible for the regulation of the sulfur amino acid pathway, requires different } \\
\text { combinations of the auxiliary factors Cbflp, Met28p, Met3Ip and Met32p }\end{array}$ \\
\hline Carbon source response & Rgtlp & 33 & $\begin{array}{l}\text { Glucose-responsive transcription factor that regulates expression of several glucose } \\
\text { transporter }(\mathrm{HXT}) \text { genes in response to glucose; transcriptional activator and } \\
\text { repressor }\end{array}$ \\
\hline
\end{tabular}


Table I: Cluster of transcription factors with GeneOntology terms related to the predicted ORFs (Continued)

\begin{tabular}{|c|c|c|c|}
\hline & Adrlp & 21 & $\begin{array}{l}\text { Required for transcription of the glucose-repressed gene ADH2, of peroxisomal } \\
\text { protein genes, and of genes required for ethanol, glycerol, and fatty acid utilization }\end{array}$ \\
\hline & Azflp & 15 & $\begin{array}{l}\text { Involved in induction of CLN3 transcription in response to glucose; genetic and } \\
\text { physical interactions indicate a possible role in mitochondrial transcription or } \\
\text { genome maintenance }\end{array}$ \\
\hline $\mathrm{pH}$ stress response & Nrglp & 28 & $\begin{array}{l}\text { Recruits the Cyc8p-Tup Ip complex to promoters; mediates glucose repression and } \\
\text { negatively regulates a variety of processes including filamentous growth and alkaline } \\
\mathrm{pH} \text { response }\end{array}$ \\
\hline Phosphate response & Pho4p & 20 & $\begin{array}{l}\text { Binds cooperatively with Pho2p to the } \mathrm{PHO} 5 \text { promoter; function is regulated by } \\
\text { phosphorylation at multiple sites and by phosphate availability }\end{array}$ \\
\hline DNA Damage & Rphlp & 17 & $\begin{array}{l}\text { JmjC domain-containing histone demethylase which can specifically demethylate } \\
\text { H3K } 36 \text { tri- and dimethyl modification states; transcriptional repressor of PHRI; } \\
\text { Rph I p phosphorylation during DNA damage is under control of the MECI-RAD53 } \\
\text { pathway }\end{array}$ \\
\hline
\end{tabular}

expression of several HXT glucose transporter genes in response to glucose), 28 for Nrg1p (mediates glucose repression and negatively regulates a variety of processes including filamentous growth and alkaline $\mathrm{pH}$ response), 21 for Adr1p (peroxisomal protein genes and genes required for ethanol, glycerol, and fatty acid utilization), 20 for Pho4p (phosphorylation at multiple sites and by phosphate availability), and 06 for Yap1p (required for oxidative stress tolerance; mediates resistance to cadmium). From this analysis, the TFs dataset is estimated to be a group that us likely to have a major influence on the extracellular secretome.

The relationship between the transcriptional regulators and predicted extracellular proteome has great complexity. Therefore, to create an $a b$ initio model, the data were shaped by graph theory. One of the graph representations was a square-directed non-weighted adjacency matrix, with 134 rows and columns. Among them, 109 were the predicted proteins identified in this study, 25 with their related TFs. The graph was created with 134 nodes and 884 edges. As illustrated in Figure 4, a three-spanning tree was extracted to illustrate the complexity of the regulatory network for each predicted ORFs. Three well-known extracellular proteins in $K$. lactis were use, along with $\alpha$-factor mating pheromone (KLLA0E19075g), invertase (KLLA0A10417g), and acid phosphatase precursor (KLLA0A00176g). Supporting material can be found at our website, http://www.yeastmolphys.ufv.br/klactis.

\section{Discussion}

Because of its distinctive physiological properties, $K$. lactis has become an important model as a non-Saccharomyces yeast. In addition, $K$. lactis has great potential for biotech-

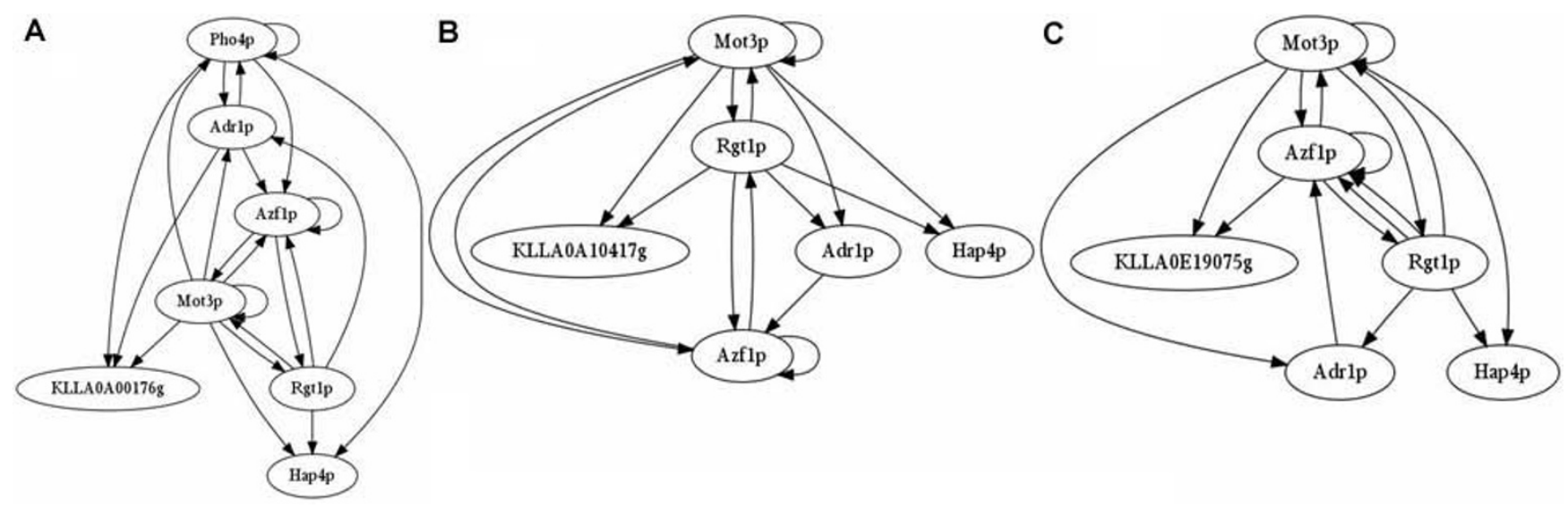

Figure 4

Spanning trees of the carbon and aerobic response of the predicted transcriptional regulatory networks of (A) acid phosphatase precursor (KLLA0A00 I 76g) (B) Invertase (KLLA0E I 90 I7); (C) $\alpha$-factor mating pheromone (KLLAOE 19075g); Transcriptional factors are represented by the small ellipses and target ORFs by larger ellipse. The edges are the presence of TFBSs in putative promoter region. 
nological applications including expression of heterologous proteins [2]. These possibilities motivated us to study the global extracellular proteome and correlate it to TFs using a bioinformatics approach. The final results have shown 109 proteins that are potentially secreted by $K$. lactis. In addition to using the TMHMM and TargetP algorithsm used by Lee et al [10] and Swaim et al [22], the Phobius [19] and WoLF PSORT [21] were applied to find transmembrane domains and subcellular addressing that would direct targeted proteins to organelles such as the endoplasmic reticulum, golgi, and proteasomes. The WOLF PSORT algorithm appeared to be more accurate; also, when the dataset of secreted proteins detected experimentally by Swain et al [22] was compared by the predicting methods of Lee et al [10], it has detected more proteins (37) than WoLF PSORT (33). However, analysis of the prediction error rate was $69.3 \%$ for WoLF PSORT and about $79.2 \%$ for TargetP. The appearance of proteins in the medium changes in different physiological conditions [24], so the predictive methods chosen here decrease error rates and improve the chances of obtaining an actual extracellular protein in a given physiological condition. The error reduction may come from the incremented algorithmic Phobius [19] combining transmembrane topology and signal peptide prediction, and the new algorithm WOLF PSORT [21] to predict the subcellular localization of proteins on the basis of their amino acid sequences using $k$-NN (k-nearest neighbor). As described by Swain $e t$ al [22], in the signal peptide detection step, the prediction algorithm SignalP v3.0 [11] was used to give two NN prediction scores, mean $\mathrm{S}$ and mean $\mathrm{D}$, and one HMM score. These NN scores were used for statistical analysis in the first step to identify extracellular proteins by the conserved secretory pathway features of a signal peptide and a signal peptidase cleavage site [10]. Accuracy in identifying extracellular proteins may be decreased because proteins that act in the periplasmic space or the cell wall also pass through the GPS. Motifs or conserved addresses for the perisplasmic space or cell wall have not yet been found. Thus, the strategy adopted to classify the results in this study focused on annotation terms and on PFam, a database of conserved domains and families [25]. The Genolevure third release is the main publicly available annotation dataset for $K$. lactis sequences. Therefore, the PFam [25] database was used in addition to updating the Genolevures annotation. Both showed five K. lactis annotated secreted proteins: acid phosphatase, repressible acid phosphatase precursor, guanosine diphosphatase, exo1,3-beta-glucanase and invertase. Although some of these proteins have not been described as acting in the extracellular space according to Domínguez et al [26], S. cerevisiae proteins are not found free in the extracellular medium but are retained in the periplasmic space or associated with the cell wall. $K$. lactis, however, does not seem to have the same characteristic; in fact, it has been reported to secrete high molecular weight proteins [1]. Thus, in this study, proteins from the periplasmic space or associated with the cell wall have been considered as part of the potential extracellular proteins dataset.

Bioinformatics identifications are probabilistic in nature, so the advantage of our analysis lies in the low cost and high speed with which these identifications can be obtained $[27,28]$; hence, this analysis exploited an ab initio model of physiological inference. The model was created using the computational extracellular proteome dataset, the transcriptional regulators repertoire mined by Bussereau et al [18], and the Yeastract methodology created by Teixeira et al [23] http://www.yeastract.com. Since gene expression programs depend on recognition of specific promoter sequences by transcriptional regulatory proteins [18], we decided to analyze the relationship between the consensus sequences or DNA binding motifs and transcriptional regulators. One of the first changes that occurs in a cell after an environmental stimulus is the content of transcriptional regulators [24]. When a set of $S$. cerevisiae transcriptional regulators orthologues and their related DNA motifs binding sites was identified, a high level of polymorphism, or DNA binding factors capable of binding to both specific and nonspecific sequences, was observed [23,29]. Because of the complex relation between TFs and the predicted secretome, the data obtained was analyzed using graph theory [24]. The empirical model may suggest many conditions that have not yet been thought of by intuitive inference. The GO terms described for each TF dataset showed possible major interactions related to stress and the cell cycle. The results of this study are in accordance with the literature, because expressions of extracellular proteins increase in stress situations or in the exponential phase when the cell requires proteins that interact in the cell wall or in the periplasmic space [1]. However, for a good secretion system, a few different proteins that can show high expression and secretion are needed. An ab initio model allows searching for both these proteins and the environmental conditions that might improve their expression and secretion.

\section{Conclusion}

Based on selected algorithms SignalP v3, Phobius, bigPIpredictor and Wolf PSORT, and adopting the highest Wolf PSORT $k$-NN scores and using multivariate T-square analysis for verification, we predicted an extracellular $K$. lactis secretome of 109 proteins. The well-known extracellular $K$. lactis proteins such as $\alpha$-factor mating pheromone, invertase, and acid phosphatase precursor were among the 109 predicted proteins. In addition, by considering the Genolevure annotations and comparing to PFam, $48 \%$ of the known proteins had enzyme activity. By applying the S. cerevisiae Yeastract database, 65 transcription 
factor orthologues were found, 23 of which had binding sites in the promoters of the 109 predicted $K$. lactis secretome. An ab initio model of physiological inference is presented. The model is a graph with 134 nodes and 884 edges that suggests a large number of relationships between the proteins and physiological conditions that can be experimentally validated. Most of the predicted TF for extracellular proteins are related to stress responses, such as drug, acid and heat resistance, as well as nitrogen limitation, which may prove useful for inducing maximal expression of the potential extracellular proteins. A condition that favors secretion could be used to design a system to improve the secretion of a desired protein. our model is stored in a public database http://www.yeastmol phys.ufv.br/klactis.

\section{Methods \\ Data Sets}

The main dataset analyzed in this study was in two files in FASTA format. Both files contained $5076 \mathrm{~K}$. lactis nucleotide and aa sequences. These data are available in the $K$. lactis third public release from the Génolevures consortium http://cbi.labri.fr/Genolevures.

To test the criteria for extracellular proteins, a validation set consisting of 95 non-redundant yeast extracellular proteins sequences (YEP) and 95 nonredundant $K$. lactis random sequences (KLRS) was assembled. The YEP dataset was obtained by searching in the UniProt protein database http://www.uniprot.org. The KLRS was assembled using a random number generator and a sequence seeker algorithm. Another validation dataset was manually extracted from Swain et al [22], consisting of $81 \mathrm{~K}$. lactis extracellular proteins identified by mass spectrometry analysis (EPMS).

The K. lactis TF dataset used in this study was from Bussereau et al [18]. The retrieved data were composed of 102 TFs identified as orthologues of $S$. cerevisiae transactivators.

\section{Algorithms and Strategy}

The entire K. lactis predicted proteins dataset was applied to SignalP v3.0 http://www.cbs.dtu.dk/services/Sig nalP $[11]$ to identify $\mathrm{N}$-terminal signal peptides. To define a positive SignalP hit, the following simultaneous criteria were used: (a) signal peptide predicted by SignalP NN with the scores mean S and mean $\mathrm{D}$; (b) signal peptide predicted by SignalP HMM considering the value of probability, and (c) signal peptide cleavage site located 10-40 aa from the N-terminus.

The group of predicted ORFs that encoded sequences with $\mathrm{N}$-terminal signal peptides was analyzed according to the three additional characteristics of transmembrane domain, GPI modification site predicted by Phobius http://phobius.sbc.su.se[19], and PI-predictor http:// mendel.imp.ac.at/gpi/gpi server.html[20]; the subcellular location was estimated using WOLF PSORT http:// wolfpsort.org[21] to identify signal addressing for subcellular locations. The obtained dataset comprised all sequences of deduced proteins potentially acting in extracellular space. The outcome set was analyzed by the PFam database http://www.sanger.ac.uk/Software/Pfam[25] in order to update the Genolevure annotations.

To correlate the computational extracellular proteome and the TF repertoire, a supporting algorithm was created on the basis of ANSI/ISO C++ strings operations [30] in the $K$. lactis chromosomes dataset. This retrieved one $\mathrm{kb}$ upstream sequence as the putative promoter region from each predicted extracellular ORF. The recovered dataset is stored in a FASTA file with the relevant identification. The relationship between this computational extracellular proteome and the transcriptional regulators repertoire was made according to Yeastract [23]. The Yeastract web tools http://www.yeastract.com and database were used to find associated TFBSs in S. cerevisiae. A second supporting $\mathrm{C}++[30]$ algorithm was created to remove S. cerevisiae TFs nonhomologous to K. lactis. The Graphviz (Graph Visualization Software, http://www.graphviz.org) package was used to draw the graph, and the spanning trees operations were implemented by Boost library 1.36 http:// www.boost.org.

\section{Statistical Analysis}

Multivariate analysis of variance was applied to verify the accuracy and determine the error rate of the computational secretome. The SignalP NN scores (mean S and D) and SignalP HMM probability were used as values in statistical analysis to determine the matrices of variance-covariance of the predicted and validations sets, and the Hotelling $\mathrm{T}^{2}$ multivariate test [31] was applied to calculate the probability of equality of the means vectors.

\section{Authors' contributions}

FP conceived and designed the project. OB developed and coded the support algorithms. LF analyzed the annotation of the transcriptional factors. CC performed the statistical analysis. All authors read and approved the final manuscript.

\section{Acknowledgements}

We thank the Brazilian Agency FAPEMIG (Fundação de Amparo à Pesquisa do Estado de Minas Gerais) for financial support.

\section{References}

I. Becerra M, Prado SD, Siso MIG, Cerdán ME: New secretory strategies for Kluyveromyces lactis $\beta$-galactosidase. Protein Engineering 200I, I 4:379-386.

2. Bolotin-Fukuhara M, Toffano-Nioche C, Artiguenave F, DuchateauNguyen G, Lemaire M, Marmeisse R, Montrocher R, Robert C, Ter- 
mier M, Wincker P, Wésolowski-Louvel M: Genomic Exploration of the Hemiascomycetous Yeasts: I I. Kluyveromyces lactis. FEBS Letters 2000, 487:66-70.

3. Tzung KW, Williams RM, Scherer S, Federspiel N, Jones T, Hansen N, Bivolarevic V, Huizar L, Komp C, Surzycki R, Tamse R, Davis RW, Agabian N: Genomic evidence for a complete sexual cycle in Candida albicans. Proc Natl Acad Sci USA 200I, 98:3249-3253.

4. Cliften P, Sudarsanam P, Desikan A, Fulton L, Fulton B, Majors J, Waterston R, Cohen BA, Johnston M: Finding functional features in Saccharomyces genomes by phylogenetic footprinting. Science 2003, $301: 71-76$.

5. Kellis M, Patterson N, Endrizzi M, Birren B, Lander ES: Sequencing and comparison of yeast species to identify genes and regulatory elements. Nature 2003, 423:241-254.

6. Kellis M, Birren BW, Lander ES: Proof and evolutionary analysis of ancient genome duplication in the yeast Saccharomyces cerevisiae. Nature 2004, 428:617-624.

7. Ramezani-Rad M, Hollenberg CP, Lauber J, Wedler H, Griess E, Wagner C, Albermann K, Hani J, Piontek M, Dahlems U, Gellissen G: The Hansenula polymorpha (strain CBS4732) genome-sequencing and analysis. FEMS Yeast Res 2003, 4:207-2I5.

8. Dietrich FS, Voegeli S, Brachat S, Lerch A, Gates K, Steiner S, Mohr C, Pöhlmann R, Luedi P, Choi S: The Ashbya gossypii genome as a tool for mapping the ancient Saccharomyces cerevisiae genome. Science 2004, 304:304-307.

9. Emanuelsson O, Nielsen H, Brunak S, Heijne S: Predicting subcelIular localization of proteins based on their $\mathbf{N}$-terminal amino acid sequence. J Mol Biol 2000, 300:1005-1016.

10. Lee SA, Wormsley S, Kamoun S, Lee AFS, Joiner K, Wong B: An analysis of the Candida albicans genome database for soluble secreted proteins using computer-based prediction algorithms. Yeast 2003, 20:595-6I0.

II. Bendtsen DJ, Nielsen H, Heijne G, Brunak S: Improved prediction of signal peptides: SignalP 3.0. J Mol Biol 2004, 340:783-795.

12. Emanuelsson $\mathrm{O}$, Brunak S, Heijne $\mathrm{G}$, Nielsen $\mathrm{H}$ : Locating proteins in the cell using TargetP, SignalP, and related tools. Nature Protocols 2007, 2:953-97I.

13. Chekmenev DS, Haid C, Kel AE: P-Match: transcription factor binding site search by combining patterns and weight matrices. Nucleic Acids Research 2005:W432-W437.

14. Heinemeyer T, Wingender E, Reuter I, Hermjakob H, Kel A, Kel OE, Ignatieva E, Ananko O, Podkolodnaya F, Kolpakov N: Databases on Transcriptional Regulation: TRANSFAC, TRRD, and COMPEL. Nucleic Acids Res 1998, 26:364-370.

15. Wingender E, Chen X, Hehl R, Karas H, Liebich I, Matys V, Meinhardt T, Prüss M, Reuter I, Schacherer F: TRANSFAC: an integrated system for gene expression regulation. Nucleic Acids Res 2000, 28:316-9.

16. Wingender E, Chen X, Fricke E, Geffers R, Hehl R, Liebich I, Krull M, Matys $\mathrm{V}$, Michael $\mathrm{H}$, Ohnhäuser R: The TRANSFAC system on gene expression regulation. Nucleic Acids Res 200I, 29:28I-3.

17. Kel AE, Gössling E, Reuter I, Cheremushkin E, Kel-Margoulis OV, Wingender E: MATCH: A tool for searching transcription factor binding sites in DNA sequences. Nucleic Acids Res 2003, 3 I (13):3576-9.

18. Bussereau F, Casaregola S, Lafay JF, Bolotin-Fukuhara M: The Kluyveromyces lactis repertoire of transcriptional regulators. FEMS Yeast Res 2006, 6(3):325-35.

19. Käll L, Krogh A, Sonnhammer ELL: A Combined Transmembrane Topology and Signal Peptide Prediction Method. Journal of Molecular Biology 2004, 338(5): 1027-1036.

20. Eisenhaber B, Bork P, Eisenhaber F: Sequence properties of GPIanchored proteins near the omega-site: constraints for the polypeptide binding site of the putative transamidase. Protein Engineering 1998, 12: I155-I I6I.

21. Horton P, Park KJ, Obayashi T, Fujita N, Harada H, Adams-Collier CJ, Nakai K: WoLF PSORT: protein localization predictor. Nucleic Acids Res 2007, 35:W585-7.

22. Swaim CL, Anton BP, Sharma SS, Taron CH, Benner JS: Physical and computational analysis of the yeast Kluyveromyces lactis secreted proteome. Proteomics 2008, 8:27|4-2723.

23. Teixeira MC, Monteiro P, Jain P, Tenreiro S, Fernandes AR, Mira NP, Alenquer M, Freitas AT, Oliveira AL, Sá-Correia I: The YEASTRACT database: a tool for the analysis of transcription regulatory associations in Saccharomyces cerevisiae. Nucleic Acids Res 2006, 34:D446-5I.
24. Babu MM, Luscombe NM, Aravind L, Gerstein M, Teichmann SA: Structure and evolution of transcriptional regulatory networks. Current Opinion in Structural Biology 2004, I 4:283-29I.

25. Finn RD, Tate J, Mistry J, Coggill PC, Sammut JS, Hotz HR, Ceric G, Forslund K, Eddy SR, Sonnhammer EL, Bateman A: The Pfam protein families database. Nucleic Acids Research 2008 , 36:D281-D288.

26. Domínguez A, Fermiñán E, Sánchez M, González FJ, Pérez-Campo FM, García S, Herrero AB, San Vicente A, Cabello J, Prado M: Non-conventional yeasts as hosts for heterologous protein production. Int Microbiol 1998, I(2): I 3 |-42.

27. Chen $Y, Y u$ P, Luo J, Jiang $Y$ : Secreted protein prediction system combining CJ-SPHMM, TMHMM, and PSORT. Mammalian Genome 2003, I 4(I 2):859-865.

28. Klee EW, Carlson DF, Fahrenkrug SC, Ekker SC, Ellis LBM: Identifying secretomes in people, pufferfish and pigs. Nucleic Acids Research 2004, 32(4): |4|4-|42|.

29. Fischer G, Rocha EPC, Brunet F, Vergassola M, Dujon B: Highly Variable Rates of Genome Rearrangements between Hemiascomycetous Yeast Lineages. PLoS Genetics 2006, 2(3):e32.

30. Stroustrup B: C++ Programming Language. In AT\&T Labs Murray Hill, New Jersey. Addison-Wesley; 2004

3I. Hotelling H: The generalization of Student's ratio. Ann Math Statist 1931, 2:360-378.
Publish with Biomed Central and every scientist can read your work free of charge

"BioMed Central will be the most significant development for disseminating the results of biomedical research in our lifetime. "

Sir Paul Nurse, Cancer Research UK

Your research papers will be:

- available free of charge to the entire biomedical community

- peer reviewed and published immediately upon acceptance

- cited in PubMed and archived on PubMed Central

- yours - you keep the copyright
BioMedcentral 